\title{
Knowledge, attitudes and practices regarding TB infection control among health science students in a TB-endemic setting
}

\author{
H van der Westhuizen', JCB Kotze 1 , H Narotam 1 , A von Delft', B Willems ${ }^{1}$, A Dramowski ${ }^{3}$ \\ 1. Division of Community Health, Stellenbosch University, South Africa \\ 2. School of Public Health and Family Medicine, University of Cape Town, South Africa \\ 3. Department of Paediatrics and Child Health, Division of Paediatric Infectious Diseases, \\ Stellenbosch University, South Africa
}

doi: 10.3396/IJIC.v11i4.030.15

\begin{abstract}
Medical and physiotherapy students in tuberculosis (TB)-endemic settings are at high risk of developing occupational TB. Didactic lectures are the traditional method for delivering education on the topic of TB infection control (TB-IC) to undergraduate health science students. The aim of this study was to evaluate the impact of a novel educational package for teaching TB-IC to undergraduate health science students in a TBendemic setting.

Undergraduate medical and physiotherapy students at Stellenbosch University, South Africa $(n=326)$ participated in a cross-sectional survey documenting TB-IC knowledge, attitudes and practices at the training institution. A self-administered questionnaire was completed before and after a novel educational intervention which utilized the health belief model (and included personal testimonials from healthcare workers and an IC expert who had survived occupational TB).
\end{abstract}

Students perceived themselves to be at high risk of occupational TB, but underestimated drug-resistant TB mortality. Pre-intervention knowledge of TB-IC measures was poor, but improved post-intervention (58\% vs

\section{Corresponding Author}

Helene-Mari van der Westhuizen

Division of Community Health, Stellenbosch University, South Africa

Email: helene1mari@gmail.com 
$78 \%$ [p < 0.001]). Senior students had better pre-intervention knowledge of N95 respirator use. Negative attitudes and TB-IC practices of senior staff at the training institution reportedly influenced those of students. TB-IC measures (natural and mechanical ventilation; airborne precaution signage; patient isolation) were reportedly poorly implemented. Access to personal protective equipment was problematic with $49 \%(87 / 177)$ of senior students stating that N95 respirators were never available.

In conclusion, students lack knowledge of TB-IC measures and report poor implementation of TB-IC measures at their training institution. A novel structured educational intervention increased students' knowledge of TB-IC measures and heightened awareness of occupational TB risk.

Keywords: Tuberculosis; Infection control; Health knowledge, attitude, practices; Occupational diseases; Prevention and control; Education, medical

\section{Introduction}

High rates of occupational TB disease are reported in a systematic review of TB in healthcare workers (HCW) from low- and middle-income countries. ${ }^{1}$ In South Africa, which has one of the highest TB incidence rates globally, ${ }^{2} \mathrm{HCW}$ are three times more likely to develop drug-sensitive (DS-TB) and six times more likely to contract drug-resistant TB (DR-TB) than the general population. ${ }^{3}$ Health science students training in these high burden countries are also at high risk of occupational TB exposure, infection and disease, although limited data are reported. ${ }^{4,5}$

Several simple and effective TB-IC measures can be used in low resource settings to reduce HCW TB exposure, including early TB diagnosis, segregation of infectious TB patients, improved natural ventilation and education of HCW. ${ }^{6}$ However in most endemic settings (including South Africa), TB-IC implementation is poor and few HCW take steps to protect themselves from TB infection, despite perceiving themselves to be at increased risk of TB disease. ${ }^{7,8}$ Improved access to information on TB infection risk has been shown to influence HCW TB-IC practices and behaviours. ${ }^{9}$ We measured the impact of a novel educational intervention on medical and physiotherapy students' awareness of TB infection risk and knowledge of TBIC and documented students' experience of TB-IC practice at their training institution.

\section{Methods}

\section{Study population, design and statistical analysis}

A convenience sample $(n=326)$ of undergraduate Medicine $(n=259)$ and Physiotherapy $(n=67)$ students in their $2^{\text {nd }}$ to $4^{\text {th }}$ years of training at Stellenbosch University, Cape Town, South Africa participated in a cross-sectional survey of TB-IC knowledge, attitudes and practices. Four "extra-curricular" novel TB educational sessions (named "TB Proof") were held between March 2012 and February 2013. Students completed a questionnaire before and after attending the educational intervention. TB and TB-IC knowledge (11), attitudes (8) and practices (18), were measured using multiple choice, true/false, Likert scale and open-ended questions. Students were stratified by year of study; only students in their clinical years ( $3^{\text {rd }}$ and $4^{\text {th }}$ years) answered questions relating to TB-IC practices $(n=191)$. Some respondents omitted answers resulting in varying sample sizes between questions. Two-way mixed model repeated measures ANOVA was conducted to compare ordinal measurements between two time points, controlling for different student groupings (pre-clinical vs clinical; medical vs physiotherapy students). Categorical variables were compared using cross-tabulation and the McNemar test (Statistica version 12).

\section{Design of the novel educational intervention: "TB Proof"}

The educational intervention followed the health belief model (Hochbaum and Rosenstock, 1958). ${ }^{10}$ The six components of the model were adapted (Table I) to influence students' desire to avoid contracting TB and 
Table I. Use of the health belief model to design the TB-IC intervention

\begin{tabular}{ll} 
Concept & Application in intervention \\
\hline Perceived & Students were provided with research estimates of occupational TB disease incidence \\
among HCW compared to the general population. ${ }^{13,11}$ Personal testimonials of HCW \\
who contracted occupational TB were shared to counter the misconception that only \\
immune-compromised people develop TB. Students could relate to their fellow HCW \\
experiences of the devastating effects of occupational TB. An example of the personal \\
account of MDR-TB delivered by Dr Dalene von Delft can be viewed at http://vimeo. \\
com/68358500. \\
\hline Perceived Severity & $\begin{array}{l}\text { Data from research reports } \\
\text { the extremely high mortality of MDR and XDR-TB (30-74\%) and explaining the side } \\
\text { effects of DR-TB treatment like nausea, vomiting, hearing loss, gout, depression and } \\
\text { social isolation. }\end{array}$ \\
\hline Perceived Benefits & $\begin{array}{l}\text { The concept of the hierarchy of TB-IC was introduced as an effective tool to reduce } \\
\text { TB infection risk: including practical suggestions on use of administrative controls } \\
\text { (separating infectious patients, introducing cough hygiene), environmental controls } \\
\text { (improving natural ventilation) and a demonstration of how to fit personal protective } \\
\text { equipment (N95 respirators). }\end{array}$ \\
\hline Perceived Barriers & $\begin{array}{l}\text { The stigma and discomfort of wearing a respirator and the negative attitudes of } \\
\text { senior clinicians towards TB- IC were discussed as possible barriers to TB-IC } \\
\text { implementation. }\end{array}$ \\
\hline The intervention suggested using the mental cue of a coughing patient as a reminder \\
to evaluate TB-IC.
\end{tabular}

build confidence in the effectiveness of the proposed action (better TB-IC implementation). The intervention consisted of personal testimonials from HCW who had survived TB and DR-TB and interactive discussions of TB-IC measures available to students, led by a TB-IC expert. The 45-minute session concluded with a call to action for students to become "agents of change" for improved TB-IC at their training institution. Students posed for a class photo wearing N95 respirators, which was uploaded to Facebook as a reminder of their commitment to implement TB-IC (www.facebook. com/TBProof). Ethical approval was obtained from the Stellenbosch University Health Research Ethics Committee (S12/06/157) and participants provided individual written informed consent.

\section{Results}

\section{Knowledge of TB and TB-IC}

Students perceived themselves to be at high risk of occupational TB (pre-intervention mean of 4.4 times increased risk compared to the general population) but adjusted this estimate downwards to a mean of 4.0 times increased risk post-intervention $(p<0.001)$. Students revised their initial estimation of the 5 -year mortality rate of a HCW with DR-TB from a mean of $32 \%$ to $44 \%$ at the post-intervention assessment ( $p$ $<0.001)$.

I am definitely more aware of the risks involved both to myself and my family. I did not know that the mortality rate was that high for drug-resistant TB.' - Participant 15 
Table II. Students' perceptions of responsibility for TB risk reduction

\begin{tabular}{lcrr}
\hline $\begin{array}{l}\text { "Whose responsibility is it to prevent health } \\
\text { sciences students at Stellenbosch University } \\
\text { from contracting TB?" }\end{array}$ & $\begin{array}{r}\text { Pre-intervention } \\
(\mathrm{n}=326)\end{array}$ & $\begin{array}{r}\text { Post-intervention } \\
(\mathrm{n}=326)\end{array}$ & P-value \\
(Students could select multiple options) & & & \\
\hline Student & $85 \%(278 / 326)$ & $92 \%(299) / 326)$ & $\mathrm{p}=0.01$ \\
\hline Hospital (the training institution) & $79 \%(257 / 326)$ & $69 \%(225 / 326)$ & $\mathrm{p}=0.01$ \\
\hline Clinician (the hospital staff) & $48 \%(155 / 326)$ & $27 \%(87 / 326)$ & $\mathrm{p}<0.001$ \\
\hline Stellenbosch University & $66 \%(216 / 326)$ & $34 \%(110 / 326)$ & $\mathrm{p}<0.001$ \\
\hline Health Professions Council of South Africa & $55 \%(180 / 326)$ & $13 \%(41 / 326)$ & $\mathrm{p}<0.001$ \\
\hline
\end{tabular}

Knowledge of N95 respirator use improved significantly from $58 \%$ (pre-intervention) to $78 \%$ (post-intervention) [p $<0.001$ ], with senior students achieving highest pre-intervention scores. Students were uncertain who was responsible for preventing TB acquisition among health science students. The question clarified that 'responsibility' included 'the provision of personal protective equipment, infection control measures and the creation and implementation of prevention policies.' Participants' responses changed significantly post-intervention, shifting assignment of responsibility away from the training institution and university, and allocating greater responsibility to the individual student (Table II).

\section{Attitudes to TB-IC}

Students reported themselves as the group least familiar with TB-IC protocols (38\%), but felt that most nurses $(58 \%)$ and doctors (51\%) were aware of, but did not follow, TB-IC protocols (Table III). When faced with a scenario of having to clerk a patient with suspected DRTB without adequate respiratory protection: students who would refuse to clerk the patient increased from $83 \%(266 / 321)$ to $93 \%(298 / 321)$ post-intervention ( $p$ $<0.001)$. Students reported the following factors that made it difficult to protect themselves: $73 \%$ of students indicated that 'superiors give the order'; $69 \%$ said 'the patient deserves care' and $66 \%$ indicated they would 'worry about their examination scores being influenced'.

\section{Table III. Students' perceptions of TB-IC attitudes and practices at the training institution}

\begin{tabular}{|c|c|c|c|}
\hline & $\begin{array}{r}\text { "....are familiar with } \\
\text { TB infection control } \\
\text { measures and act } \\
\text { accordingly." }\end{array}$ & $\begin{array}{r}\text { "...are familiar with } \\
\text { TB infection control } \\
\text { measures and do not act } \\
\text { accordingly." }\end{array}$ & $\begin{array}{r}\text { "...are not familiar with } \\
\text { TB infection control } \\
\text { measures and do not } \\
\text { enact any protocols." }\end{array}$ \\
\hline "Doctors..." & $\begin{array}{r}44 \% \\
(79 / 179) \\
\end{array}$ & $\begin{array}{r}51 \% \\
(91 / 179) \\
\end{array}$ & $\begin{array}{r}5 \% \\
(9 / 179) \\
\end{array}$ \\
\hline "Nursing staff..." & $\begin{array}{r}33 \% \\
(59 / 178) \\
\end{array}$ & $\begin{array}{r}58 \% \\
(103 / 178) \\
\end{array}$ & $\begin{array}{r}9 \% \\
(16 / 178) \\
\end{array}$ \\
\hline "Fellow students..." & $\begin{array}{r}18 \% \\
(32 / 180)\end{array}$ & $\begin{array}{r}44 \% \\
(79 / 180)\end{array}$ & $\begin{array}{r}38 \% \\
(69 / 180)\end{array}$ \\
\hline
\end{tabular}


I was in denial about my risk of contracting TB as a medical student. I didn't realize that my risk was actually that high. I will never again take working with TB patients lightly. I used to think that because I am healthy I will not contract TB or show signs and symptoms of TB. [I will] demand protection when seeing patients suspected of having TB [and] refuse to see these patients if no protection is available.' Participant 2

As part of the pre-intervention questionnaire, students were asked how a personal diagnosis of TB would impact on their quality of life. Most respondents $(64 \% ; 206 / 321)$ indicated it would have a major effect, $30 \%(97 / 321)$ a moderate effect, $5 \%(17 / 321)$ a small effect and $<1 \%(1 / 321)$ no effect. The same question was posed for DR- TB, with most respondents, 93\% (299/321) reporting it would have a major effect.

'The intervention made me more aware of the risk of TB and how it can impact the person's life.' Participant 11

\section{TB-IC practices}

\section{Administrative controls:}

Coughing patients were seen very frequently (three times a week and more) by $72 \%(130 / 180)$ of respondents. Untreated patients with signs and symptoms suggestive of active TB were seen by $60 \%$ of respondents (109/182) on a frequent basis (once a week or more). Patients who had discontinued TB treatment were seen frequently (once a week or more) by $63 \%$ (114/182) of respondents. Respiratory hygiene was reportedly poorly implemented, with low availability of surgical masks to place on coughing patients; only 51\% (92/182) reported that surgical masks were frequently or always available. Students reported low utilization of patient isolation with $52 \%$ indicating that the hospital was 'not effective' or 'only marginally effective' at isolating patients with confirmed DR-TB (Table IV). Infection control 'airborne transmission' notification signs were underutilized (consistently used by only 22\%, 39/178). Only 25\% (44/178) reported that doors to TB isolation rooms were kept closed.

\section{Environmental controls:}

Natural ventilation (open windows) was infrequently encountered in the training institution $(60 \%$ reported windows were always closed; 108/179). The most frequently reported reasons for this practice were that the windows could not be opened (36\%); nursing staff did not support the open window policy (33\%) and that patients would get cold (24\%). Mechanical ventilation was reportedly not used $(55 \%$; 90/164) with respondents indicating that it was not present $(32 \%)$, they did not know how it was operated $(26 \%)$ or that it was broken (22\%).

Table IV. Students' perceptions of TB isolation practice at the training institution

\begin{tabular}{lrrrr} 
Question & $\begin{array}{r}\text { Not effective } \\
\text { at all }\end{array}$ & $\begin{array}{r}\text { Only } \\
\text { marginally } \\
\text { effective }\end{array}$ & $\begin{array}{r}\text { Reasonably } \\
\text { effective }\end{array}$ & $\begin{array}{r}\text { Very } \\
\text { effective }\end{array}$ \\
\hline $\begin{array}{l}\text { How effective is the institution where you do } \\
\text { Clinical rotations at isolating patients with TB }\end{array}$ & $\begin{array}{r}30 \% \\
\text { symptoms? }\end{array}$ & $\begin{array}{r}37 \% \\
(90 / 179)\end{array}$ & $\begin{array}{r}12 \% \\
(66 / 179)\end{array}$ & $\begin{array}{r}(22 / 179) \\
(1 / 179)\end{array}$ \\
\hline $\begin{array}{l}\text { How effective is the institution where you do } \\
\text { Clinical rotations at isolating confirmed TB }\end{array}$ & $25 \%$ & $44 \%$ & $27 \%$ & $4 \%$ \\
patients? & $(45 / 179)$ & $(79 / 179)$ & $(48 / 179)$ & $(7 / 179)$ \\
\hline $\begin{array}{l}\text { How effective is the institution where you do } \\
\text { Clinical rotations at isolating confirmed MDR- }\end{array}$ & $22 \%$ & $30 \%$ & $32 \%$ & $16 \%$ \\
and XDR-TB patients? & $(40 / 179)$ & $(53 / 179)$ & $(58 / 179)$ & $(28 / 179)$ \\
& & & &
\end{tabular}




\section{Personal protective equipment:}

When asked whether personal protective equipment (N95 respirators) was available, 49\% (86/177) of respondents said it was never available when needed, with only $9 \%(15 / 177)$ reporting that respirators were always available.

\section{Discussion}

There is a common misconception that health science students are less likely to develop TB because of their better socio-economic and health status than the general population. ${ }^{2}$ LTBI was consistently associated with markers of occupational exposure, but in high income countries it was more often associated with non-occupational factors. The median annual incidence of TB infection attributable to health care work was $5.8 \%$ (range $0-11 \%$ ). In reality, health science students in TB-endemic settings have significant TB exposure during training (as confirmed in this study) and are at increased risk of developing occupational TB disease. Medical and physiotherapy students (and other categories of health science students) are dependent on their undergraduate curricula and clinical teaching to fully communicate the increased risk of occupational TB and equip them with the TBIC knowledge and skills needed to protect themselves.

Although pre-intervention knowledge of N95 respirator use was poor (especially among junior students), a significant increase of $20 \%$ in scores postintervention was observed. Senior students achieved higher knowledge scores pre-intervention, suggesting that a degree of education regarding respirator usage is taking place in the clinical environment. In addition to knowledge gain, students exhibited attitudinal change post-intervention. More students would refuse to see patients without the required personal protective equipment and more acknowledged personal responsibility for reducing their risk of contracting TB. Based on these three indicators that changed significantly post-intervention, the intervention was at least partly successful in increasing TB-IC knowledge and changing attitudes.

Poor levels of TB-IC implementation have been documented in South African health care facilities. Students in this study observed many inadequacies in TB-IC implementation at their training institution. Missed opportunities for administrative controls included the lack of surgical masks for respiratory hygiene and inconsistent implementation of patient isolation measures. Environmental controls (including natural and mechanical ventilation) were inadequately utilized. N95 respirators were not universally available to health science students and HCW and there was no respirator fit-testing programme for students.

Students perceived many $\mathrm{HCW}$ in their training institution to be poor role models for TB-IC best practice. Students also noted that although doctors and nursing staff had adequate TB-IC knowledge, they failed to apply these measures in clinical practice. Incorrect practices among senior colleagues appeared to adversely affect students' perceptions of the importance of TB-IC. Students also highlighted potential barriers to compliance with TB-IC protocols including being overruled by superiors and possible adverse academic consequences.

Strengths of the intervention included use of occupational TB survivors (HCW's and health science students) to provide first-hand accounts of their experiences. An infection control expert presented the guidelines and the students were given an opportunity to interactively practice donning and doffing an N95 respirator. The study was led by medical students and this formed an important part of the intervention's message: students can be active role players and change agents for improved TB-IC practices.

A limitation of the study design is that TB-IC attitudes and practices were reported and not directly observed. Knowledge gain alone cannot be directly translated to changed HCW behaviour. Additional and ongoing interventions in the clinical training environment would be required to sustain and reinforce gains from this TB-IC educational intervention. Sample selection depended on voluntary class attendance and voluntary participation. The time between the pre- and postintervention was 45 minutes, and a longer lapse is needed to test knowledge retention. The questionnaire used has not been validated as a KAP tool.

As a result of conducting and disseminating the results of this study at our institution, there have been several positive developments. A student occupational TB prevention policy has been developed, N95 respirators 
are now supplied annually to all students in conjunction with a N95 respirator fit-testing programme and the 'TB Proof' intervention has been formally incorporated into the undergraduate curricula for Stellenbosch University health science students.

The 'TB Proof' intervention was expanded to include TB advocacy and awareness activities for HCW at government and private facilities and members of the public. This educational initiative has since been delivered to an additional $6000 \mathrm{HCW}$, medical and health science students at Stellenbosch University and the University of Cape Town. Additional planned interventions include the development of a student TB screening/reporting programme and ongoing advocacy for improved financial support for students who contract occupational TB.

\section{Conclusion}

Health science students in TB-endemic settings are at increased risk of TB exposure and occupational TB disease. Improved knowledge and awareness of TB risk and TB-IC measures can be achieved using a novel educational intervention. Better TB-IC implementation and clinician role models demonstrating TB-IC best practice are likely to achieve attitudinal and behavioural change among students.

\section{Acknowledgments}

We are grateful for the funding provided by the Community Health Department, Faculty of Medicine and Health Sciences, Stellenbosch University and SURMEPI (Stellenbosch University Rural Medical Education Partnership Initiative). This research has been supported by the US President's Emergency Plan for AIDS relief (PEPFAR) through HRSA under the terms of T84HA21652). We would like to thank the TB survivors that are part of TB Proof for sharing their stories, specifically professional nurse Pat Bond, Dr Dalene von Delft and Dr Bart Willems, as well as Prof Martin Kidd for the statistical analysis. We acknowledge 3M for sponsoring N95 respirators to all participants. We thank all participants for their time and dedication to this study.

\section{References}

1. Joshi R, Reingold A, Menzies D, Pai M. Tuberculosis among healthcare workers in low- and middle-income countries: a systematic review. PLoS Med [Internet] 2006; 3(12). http:// dx.doi.org/10.1371/journal.pmed.0030494

2. World Health Organization. World Health Organisation Global Tuberculosis Report [Internet]. 2015. Available from: http://www.who.int/tb/publications/global_report/en/. [Accessed December 14, 2015]

3. O'Donnell MR, Jarand J, Loveday M, et al. High incidence of hospital admissions with multidrug-resistant and extensively drug-resistant tuberculosis among South African healthcare workers. Ann Intern Med 2010; 153: 516-522. http://dx.doi. org/10.7326/0003-4819-153-8-201010190-00008

4. Mugerwa $H$, Byarugaba D. High prevalence of tuberculosis infection among medical students in Makerere University, Kampala: results of a cross sectional study. Arch Public Heal 2013. Available from: http://link.springer.com/ article/10.1186/0778-7367-71-7. [Accessed February 5, 2015]

5. Silva VM, Cunha AJ, Oliveira JR, et al. Medical students at risk of nosocomial transmission of Mycobacterium tuberculosis. Int J Tuberc Lung Dis 2000; 4: 420-426.

6. World Health Organization. WHO policy on TB infection control in healthcare facilities, congregate settings and households. 2009. Available from: http://apps.who.int/iris/ handle/10665/44148. [Accessed July 3, 2015]

7. Farley JE, Tudor C, Mphahlele M, et al. A national infection control evaluation of drug-resistant tuberculosis hospitals in South Africa. Int / Tuberc Lung Dis 2012; 16: 82-89. http:// dx.doi.org/10.5588/ijtld.10.0791

8. van Rie A, McCarthy K, Scott L, Dow A, Venter WD, Stevens WS. Prevalence, risk factors and risk perception of tuberculosis infection among medical students and healthcare workers in Johannesburg, South Africa. S Afr Med / 2013; 103: 853-857. http://dx.doi.org/10.7196/samj.7092

9. Kanjee Z, Amico KR, Li F, Mbolekwa K, Moll P, Friedland GH. Tuberculosis infection control in a high drug-resistance setting in rural South Africa: information, motivation, and behavioral skills. J Infect Public Health 2012; 5: 67-81. http://dx.doi. org/10.1016/j.jiph.2011.10.008

10. Glanz K, Rimer BK, Lewis FM, Eds. Health Behavior and Health Education, 3rd ed. San Francisco: Jossey-Bass 2002.

11. Claassens MM, Van Schalkwyk C, Du Toit E, et al. Tuberculosis in healthcare workers and infection control measures at primary healthcare facilities in South Africa. PLoS One 2013; 8: e76272. http://dx.doi.org/10.1371/journal.pone.0076272

12. Farley JE, Ram M, Pan W, et al. Outcomes of multi-drug resistant tuberculosis (MDR-TB) among a cohort of South African patients with high HIV prevalence. PLoS One 2011; 6: e20436. http://dx.doi.org/10.1371/journal.pone.0020436

13. Pietersen E, Ignatius E, Streicher EM, et al. Long-term outcomes of patients with extensively drug-resistant tuberculosis in South Africa: a cohort study. Lancet 2014; 383: 1230-1239. http://dx.doi.org/10.1016/S0140-6736(13)62675-6 